\section{The stability of biological nomenclature: yeasts}

SIR-The strictures of Erzinclioglu and Unwin on the inconvenient instability of zoological nomenclature (Nature 320, $687 ; 1986)$ apply equally to other kinds of organism, including the yeasts. For nontaxonomists, the rapidity with which yeast names change is farcical. For example, Zygosaccharomyces fermentati' was altered to Saccharomyces cerevisiae by Lodder and Kreger-van Rij in 1952'. back to Zygosaccharomves fermentati by Kudriavzev in 1954. to Saccharomvces montanus by Phaff, Miller and Shifrine in 1956', to Torulaspora manchurica in 1975 by van der Walt and Johannsen and finally (?) back again to Zygosaccharomyces fermentati by von Arx and his colleagues in 1977".

The same process continues even now. As part of a major work on yeast taxonomy? C.P. Kurtzman gave descriptions of thirty species of the genus Hansenula, which he then proceeded to abolish in a paper published the same year".

Part of the trouble comes from the romantic confusion of biological classification with evolutionary studies, exemplified by S. J. Gould's words":

"taxonomies are not neutral hat-racks for the pristine facts of nature. They are theories that create and reflect the deep structure of science and human culture.

Too many taxonomists appear to subscribe to this kind of sentimental codswallop and seem quite unable to understand that instability of nomenclature seriously impairs the value of their work to biology as a whole. How many biologists who work with yeasts know that publications on Saccharomyces carlsbergensis, Saccharomyces uvarum, Saccharomyces cerevisiae or Saccharomyces logos might refer to the same yeast? At best, this is an inconvenience; at worst, it retards understanding of the biology of that yeast.

It would be perfectly practicable for there to be an international body, responsible for the nomenclature of each group of organisms, and briefed to give their names helpful stability.

School of Biological Sciences.

\section{J. A. BARNEI"I}

University of East Anglia,

Norwich NR4 7TJ, UK

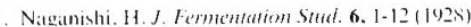

Leder. J.de Kreger-yan Rii. N.J.W. The Yeasts: “Tavo, nomic Sudt (North-Holland. Amsterdam. 1952).

Kudriarzes, V.I. Sistematika Drozhzhei (A hademii Nauh. Moseow. 1954$)$

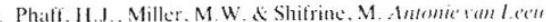

wenhock 22. 145-161 (1956).

Res. Res. Rep. No. 325 (1975).
bon Arx. J.A. Rodriguer de Mirandit. 1... Smith. .1.1. d

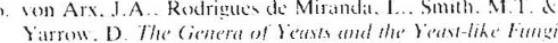

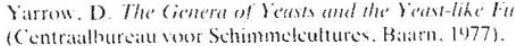

Kurt/man. C.P. in The Yousts: a Taxomomic Studs led Kreger-yan Rij. N.J.W 165-213 (Elserier. Amsterdam 105i

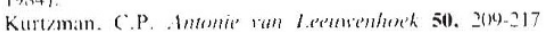

Kintsing

9. Could. S.1. Numure 313, 515-5166(1985).

\section{Microtubule assembly} in the axon

SiR-Bamburg et al. (Nature 321, 788; 1986) present a novel view of microtubule assembly in the axon. They show that axonal elongation can be inhibited by an application of microtubule depolymerizing drugs or taxol at the growth cone. The same concentrations of these drugs applied to the neurite or cell body have no effect on motility. The authors conclude that the drugs may act by inhibiting microtubule assembly at the distal tip of the axon. They also postulate that unassembled tubulin is transported along the axon to the growth cone, and there adds on to existing microtubules.

As presented, the experimental measurements of axonal length with time are interpreted as a reflection of the extent of microtubule assembly. Such measurements of extent cannot be used to distinguish between effects of the drugs on the rate constants for assembly or disassembly. The microtubule-depolymerizing drugs could act by increasing the rate of disassembly at the tip. For this alternative explanation to hold, one must postulate only that microtubules at the growth cone, but not along the neurite, are in dynamic equilibrium with a pool of monomer. There is no need to require that a net assembly reaction occurs in this region. The drugs could have their effect by shifting the dynamic equilibrium towards monomer. The existence of such an equilibrium state is a feature of other models of microtubule control in axons. For example, if microtubules are transported in the assembled state, they must be disassembled at the end of the axon. Should such an equilibrium exist, sufficiently high concentrations of drug not only might inhibit neurite extension but also cause net retraction. That outcome is reported by Bamberg et al. Thus, these data do not demonstrate microtubule assembly at the ends of axons. More direct experiments are required to demonstrate this reaction. Frank SOlOMON

Department of Biology,

Massachusetts Institute of Technology, Cambridge,

Massachusetts 02139, USA

\section{Disease gene relationship seen}

SIR-A recent article by Royer-Pokora et al.' described the isolation of a gene that is abnormal in the X-linked form of the phagocytic disorder chronic granulomatous disease (X-CGD), which is thought to be due to a lesion in the NADPH-oxidase system of these cells². An unusual $b$-type cytochrome and a flavoprotein ${ }^{+}$have been proposed as candidates for the polypeptide encoded by the Z-CGD locus, but the nucleotide sequence of the cDNA clones isolated by Royer-Pokora et al. predicted a polypeptide that showed no significant homology to known sequences in the GENBANK or protein databases. The authors could not identify any potential haem-binding region in the primary structure of the X-CGD protein.

We have, however, found a region of the predicted X-CGD sequence that is similar to the haem-binding region of the cytochrome P-450 proteins, and in particular to cytochrome $\mathrm{P}-450$ form $\mathrm{PBcl}$ from rabbit liver. The figure below illustrates the point, and identifies with an asterisk the cysteine thiolare ligand to the haem prosthetic group of the cytochrome.

$x-C G D$ protein

Cytochrome $\mathrm{P}-450$

form $\mathrm{PBCl}$

L C G P E A L A E T

$V C \star V G E$ A L A R M

The haem-binding region is highly conserved among cytochromes P-450, some of which have only a slight overall sequence similarity to one another. It is of interest that the two regions are located at equivalent positions in $\mathrm{X}-\mathrm{CGD}$ and cytochrome P-450 (at about amino acid 435).

Alan Ashworth

Elizabeth A. Shephard

Department of Biochemistry,

University College London,

London WCIE 6BT, UK

IAN R. Phillits

Departments of Pharmaceutical

Chemistry and Pharmacology,

The School of Pharmacy,

University of London,

London WCIN $1 A X$,

$U K$

1. Rover-Pokora. B. et al. Nature 322. 32-38 (1986)

2. Tauber. A.I. Borregaard. N.. Simons. E. \& Wright. J. Medicine 62, 286-309 (1983).

Segal. A.W. et al. New Engl. J. Med. 308, 245-251 (1983).

Markert. M.. Glass. G.A.. \& Barbior. B.M. Proc natn. Acad. Sci. U.S.A. 82, 31+4-3148(1985).

Leighton. J.K.. DeBrunner-Vossbrinck. B.A \& Kemper. B. Biochemistrv 23. 203-210(1984).

\section{Mankind's genetic bottleneck}

SIR-Your correspondents question whether our interpretation of a small bottleneck in human evolution based on the patterns of distribution of $\beta$-globin haplotypes is correct and. if so, whether it is relevant to the origins of mankind. Our brief answer is that. although the modern distribution of these genes can in itself tell us rather little about where and how modern Homo sapiens originated. it is nevertheless the case that - given the assumptions of our very simple model and applying it only to $\beta$-globins - passage through such a bottleneck is an inevitable implication of the present-dav distribution of $\beta$-globin variants. We attempted to root our evolutionary tree by reference to recent work on human fossils. which suggests that the most ancient $H$. sapiens are found in Africa. It is of course the case 